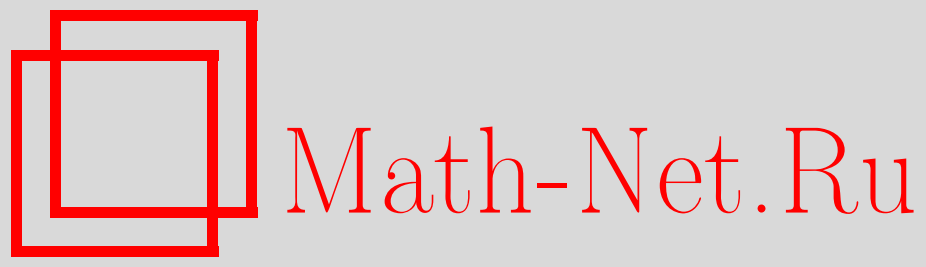

М. Брешар, Э. В. Киссин, В. С. Шульман, О йордановых идеалах и подмодулях: алгебраический и аналитический аспекты, Функи. анализ и его прил., 2008, том 42, выпуск 3, 71-75

DOI: https://doi.org/10.4213/faa2914

Использование Общероссийского математического портала MathNet.Ru подразумевает, что вы прочитали и согласны с пользовательским соглашением

http://www . mathnet.ru/rus/agreement

Параметры загрузки:

IP : 107.22 .136 .117

26 апреля 2023 г., 16:24:14

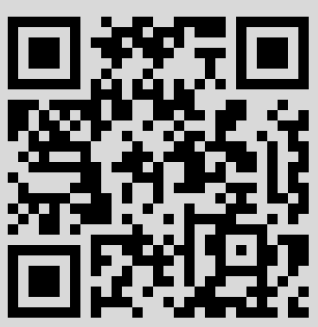




\title{
О йордановых идеалах и подмодулях: алгебраический и аналитический аспекты
}

\author{
(c) 2008. М. БрешАР, Э. В. КИССИН, В. С. ШуЛЬмАН
}

1. Введение. Пусть $\mathscr{A}$ - алгебра над полем $F$, характеристика которого не равна двум. Линейное подпространство $\mathscr{J}$ алгебры $\mathscr{A}$ называется ее йордановым идеалом, если

$$
a \circ b:=a b+b a \in \mathscr{J} \text { для всех } a \in \mathscr{A} \text { и } b \in \mathscr{J} .
$$

Во многих случаях все йордановы идеалы алгебры оказываются ее обычными (двусторонними) идеалами. Такие алгебры называются свободными от йордановых идеалов или, сокращенно, JIF-алгебрами. Начиная с фундаментальных работ Херстейна [4] и Джекобсона-Рикарта [5], изучались условия на алгебру, обеспечивающие ее принадлежность к классу JIF-алгебр. Было показано, что этот класс содержит все простые алгебры [4], все матричные алгебры $M_{n}(\mathscr{B})$, где $\mathscr{B}$ унитальна [5], все $W^{*}$-алгебры (для собственно бесконечных $W^{*}$-алгебр это было установлено в [3]; общий случай был рассмотрен в [1] и в неопубликованной работе Л. Бунса) и многие другие важные примеры.

Будем обозначать через $\operatorname{Alg}([\mathscr{A}, \mathscr{A}])$ (соответственно через $\operatorname{Id}([\mathscr{A}, \mathscr{A}]))$ подалгебру (соответственно идеал) в $\mathscr{A}$, порожденный всеми коммутаторами $[a, b]$ $=a b-b a$, где $a, b \in \mathscr{A}$. В [1] было показано, что класс JIF-алгебр содержит все алгебры, порожденные коммутаторами, т. е. алгебры, удовлетворяющие условию

$$
\mathscr{A}=\operatorname{Alg}([\mathscr{A}, \mathscr{A}]) .
$$

Пусть теперь $X$ - бимодуль над $\mathscr{A}$. Подпространство $Y$ в $X$ называется йордановым $\mathscr{A}$-подмодулем бимодуля $X$, если

$$
a \circ y:=a y+y a \in Y \text { для всех } a \in \mathscr{A} \text { и } y \in Y .
$$

Йордановы $\mathscr{A}$-подмодули самой алгебры $\mathscr{A}-$ это ее йордановы идеалы. Будем говорить, что алгебра $\mathscr{A}$ свободна от йордановых подмодулей (или, сокращенно, является JSF-алгеброй), если все йордановы $\mathscr{A}$-подмодули любого $\mathscr{A}$-бимодуля являются его подбимодулями. Оказывается, что многие условия, достаточные для включения $\mathscr{A}$ в класс JIF-алгебр, обеспечивают и более сильное свойство быть JSF-алгеброй.

Подход, основанный на рассмотрении бимодулей, более удобен технически; кроме того, будучи более общим, он имеет более широкие приложения. Так, информация о строении йордановых $\mathscr{A}$-подмодулей может быть использована для описания йордановых идеалов всех алгебр, содержащих $\mathscr{A}$.

Существуют естественные банахово-алгебраические аналоги рассмотренных выше понятий и задач. Среди имеющихся на этот счет результатов наиболее значительным является теорема Сивина-Юда [2], утверждающая, что все замкнутые йордановы идеалы произвольной $C^{*}$-алгебры являются идеалами.

Одной из причин, заставивших нас обратиться к изучению йордановых идеалов и подмодулей, явилась обнаруженная в [6] их связь с теорией операторногладких функций. Здесь важны так называемые симметрично-нормированные (с.-н.) йордановы идеалы (точное определение см. ниже). В [6] было показано, 
что в алгебрах $B(H)$ и $K(H)$ всех ограниченных и соответственно компактных операторов на гильбертовом пространстве $H$ все с.-н. йордановы идеалы являются идеалами.

В настоящей работе рассматривается как чисто алгебраический, так и банахово-алгебраический варианты. В частности, даны необходимые и достаточные условия, при которых алгебра является JSF-алгеброй. Доказано, что если $C^{*}$-алгебра не имеет мультипликативных функционалов, то все ее с.-н. йордановы идеалы являются с.-н. идеалами. Дан ответ (отрицательный) на вопрос о том, всякий ли йорданов идеал $C^{*}$-алгебры является идеалом.

2. Алгебраические результаты. Условимся говорить, что алгебра $\mathscr{A}$ обладает свойством максимальности (является тах-алгеброй), если любой собственный идеал содержится в максимальном собственном идеале. Например, если у $\mathscr{A}$ есть конечное подмножество, не содержащееся ни в каком собственном идеале, то она является тах-алгеброй. Таким образом, все унитальные, все простые и все конечно порожденные алгебры обладают свойством максимальности.

Теорема 1. Рассмотрим следующие условия на алгебру $\mathscr{A}$ :

(i) $\mathscr{A}$ является JSF-алгеброй;

(ii) $\operatorname{Id}([\mathscr{A}, \mathscr{A}])=\mathscr{A}$;

(iii) $\operatorname{Alg}([\mathscr{A}, \mathscr{A}])=\mathscr{A}$;

(iv) $\operatorname{Id}([[\mathscr{A}, \mathscr{A}], \mathscr{A}])=\mathscr{A}$.

Тогда (iv) $\Longleftrightarrow$ (iii) $\Longrightarrow$ (i) $\Longrightarrow$ (ii) .

Если $\mathscr{A}$ обладает свойством максимальности, то условия (i)-(iv) эквивалентны.

Легко видеть, что (ii) эквивалентно отсутствию в $\mathscr{A}$ идеалов с коммутативной факторалгеброй.

Поскольку для некоммутативных простых алгебр условие (ii) выполнено, из теоремы 1 вытекает следующее обобщение упомянутого во введении результата Херстейна:

Следствие 2. Все некоммутативные простые алгебры являются JSF-алгебрами.

Принимая во внимание, что унитальные коммутативные банаховы алгебры имеют мультипликативные функционалы, получаем

Следствие 3. Для унитальной банаховой алгебры кажсдое из условий (i)(iv) теоремь 1 эквивалентно отсутствию мультипликативных фучкиионалoв.

Пусть $\mathscr{G}$ - грассманова алгебра со счетным множеством $\left\{x_{1}, x_{2}, \ldots\right\}$ образующих $\left(x_{i} x_{j}+x_{j} x_{i}=0\right)$, и пусть $\mathscr{I}$ - идеал, порожденный всеми элементами вида

$$
x_{i}-x_{3 i-1} x_{3 i} x_{3 i+1}=x_{i}-\frac{1}{2}\left[x_{3 i-1}, x_{3 i}\right] x_{3 i+1}, \quad i=1,2, \ldots .
$$

Можно доказать, что $\mathscr{A}=\mathscr{G} / \mathscr{I}$ не является JSF-алгеброй и даже JIF-алгеброй. Однако $\mathscr{A}$ не имеет идеалов с коммутативным фактором. Таким образом, условие (ii) теоремы 1, вообще говоря, не влечет за собой (i).

3. Банаховы алгебры. Пусть теперь $\mathscr{A}$ - банахова алгебра. В этом случае естественно рассматривать класс банаховых $\mathscr{A}$-бимодулей, т. е. банаховых пространств $X$, являющихся $\mathscr{A}$-бимодулями, причем

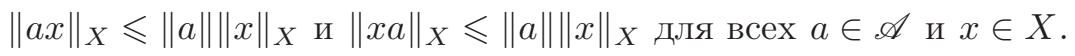


Будем говорить, что $\mathscr{A}$ топологически свободна от йордановых подмодулей (является TJSF-алгеброй), если любой замкнутый йорданов подмодуль произвольного банахова $\mathscr{A}$-бимодуля является его подбимодулем.

Ясно, что любая банахова JSF-алгебра входит в класс TJSF-алгебр. Обратное включение неверно: например, все идеалы Шэттена $C_{p}$ являются TJSF-, но не JSF-алгебрами. Однако для унитальных алгебр, как мы сейчас увидим, эти классы совпадают.

Следующая теорема является банахово-алгебраической версией теоремы 1. Отметим, что рассматриваемые в ней условия (i)-(iv) слабее соответствующих алгебраических условий из теоремы 1.

Теорема 4. Рассмотрим следующие условия на банахову алгебру $\mathscr{A}$ :

(i) $\mathscr{A}$ топологически свободна от йордановых подмодулей;

(ii) $\overline{\operatorname{Id}([\mathscr{A}, \mathscr{A}])}=\mathscr{A}$;

(iii) $\overline{\operatorname{Alg}([\mathscr{A}, \mathscr{A}])}=\mathscr{A}$;

(iv) $\overline{\operatorname{Id}([[\mathscr{A}, \mathscr{A}], \mathscr{A}])}=\mathscr{A}$;

(v) $\mathscr{A}$ не имеет мультипликативных функиионалов.

Тогда (iv) $\Longleftrightarrow(\mathrm{iii}) \Longrightarrow$ (i) $\Longrightarrow$ (ii) $\Longrightarrow(\mathrm{v})$.

Для унитальных алгебр, а такэе для всех $C^{*}$-алгебр условия (i)-(v) эквивалентны.

Вообще говоря, из (v) не следует (ii) (в качестве примера можно взять любую нильпотентную банахову алгебру). Однако для сравнительно «хороших» неунитальных алгебр вопрос открыт; более того, он эквивалентен известной проблеме существования замкнутого идеала:

Предложение 5. Пусть $\mathbb{A}$ - класс всех банаховых алгебр $\mathscr{A}$, у которых всякий замкнутый идеал содержится в максимальном замкнутом идеале. Следующие утверждения эквивалентны:

(i) импликачия (v) $\Longrightarrow$ (ii) (для условий из теоремы 4) справедлива для всех $\mathscr{A} \in \mathbb{A}$;

(ii) всякая коммутативная топологически простая банахова алгебра одномерна.

4. Симметрично-нормированные йордановы подмодули в бимодулях над $C^{*}$-алгебрами. Напомним, что идеал $J$ банаховой алгебры $\mathscr{A}$ называется симметрично-нормированнным (с.-н.), если он является банаховым пространством относительно некоторой нормы $\|\cdot\|_{J}$, причем существует число $D>0$, такое, что

$$
\|a x b\|_{J} \leqslant D\|a\|\|b\|\|x\|_{J} \text { для всех } a, b \in \mathscr{A} \text { и } x \in J .
$$

Аналогично, йорданов идеал $J$ банаховой алгебры $\mathscr{A}$ называется $c .-$. йордановым идеалом, если он наделен нормой $\|\cdot\|_{J}$, относительно которой является банаховым пространством, и при некотором $D>0$

$$
\|a \circ x\|_{J} \leqslant D\|a\|\|x\|_{J} \text { для всех } a \in \mathscr{A} \text { и } x \in J .
$$

Отметим, что если с.-н. йорданов идеал является идеалом, это еще не означает, что он является с.-н. идеалом.

Аналогично определяются с.-н. йордановы подбимодули в бимодулях над банаховыми алгебрами.

Очевидно, что все замкнутые йордановы $\mathscr{A}$-подмодули в банаховом модуле $X$ являются с.-н. йордановыми подмодулями по отношению к норме $\|\cdot\|_{X}$. Об- 
ратное, однако, неверно. Например, $B(H)$ как банахов $B(H)$-бимодуль имеет только один нетривиальный замкнутый йорданов подбимодуль $K(H)$, но, в то же время, он обладает множеством с.-н. идеалов с разнообразными алгебраическими и аналитическими свойствами.

Мы рассмотрим условия, которым должна удовлетворять алгебра $\mathscr{A}$ для того, чтобы любой с.-н. йорданов подмодуль $\left(Y,\|\cdot\|_{Y}\right)$ любого банахова $\mathscr{A}$-бимодуля являлся банаховым $\mathscr{A}$-бимодулем (относительно нормы $\|\cdot\|_{Y}$ ).

Чтобы сформулировать эти условия, нам нужен количественный вариант свойства алгебры быть порожденной коммутаторами. Пусть

$$
\mathscr{Z}(\mathscr{A})=\{[a, b]: a, b \in \mathscr{A},\|a\| \leqslant 1,\|b\| \leqslant 1\} .
$$

Будем говорить, что $\mathscr{A}$ принадлежит классу $\mathfrak{L}$, если существует такое число $t>0$, что замкнутая выпуклая оболочка мультипликативной подполугруппы в $\mathscr{A}$, порожденной $t \mathscr{Z}(\mathscr{A})$, содержит некоторый шар алгебры $\mathscr{A}$.

Класс $\mathfrak{L}$ содержит, например, все алгебры вида $M_{n}(B)$, где $B$ - произвольная унитальная банахова алгебра.

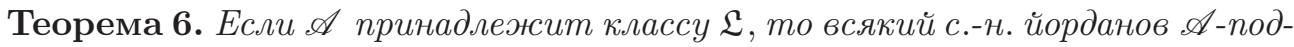
модуль любого банахова $\mathscr{A}$-бимодуля является банаховым $\mathscr{A}$-бимодулем.

Оказывается, что для $C^{*}$-алгебры принадлежность классу $\mathfrak{L}$ эквивалентна отсутствию мультипликативных функционалов. Таким образом, мы получаем следующий критерий:

Теорема 7. Для $C^{*}$-алгебры А्A следующие условия эквивалентны:

(i) $\mathscr{A}$ не имеет мультипликативных функиионалов;

(ii) все с.-н. йордановы подмодули любого банахова $\mathscr{A}$-бимодуля являются банаховыми $\mathscr{A}$-бимодулями.

Менее ясна ситуация с с.-н. йордановыми идеалами $C^{*}$-алгебр. Понятно ввиду предыдущей теоремы, что если $C^{*}$-алгебра не имеет мультипликативных функционалов, то ее с.-н. йордановы идеалы являются с.-н. идеалами. Оказывается, что в случае, когда мультипликативные функционалы существуют, с.-н. йордановы идеалы могут не быть идеалами (это тем более неожиданно, что до сих пор не было известно примеров нетривиальных йордановых идеалов $C^{*}$-алгебр).

Теорема 8. Существует $C^{*}$-алгебра $\mathscr{A}$, являющаяся коммутативньм расширением алгебры $K(H)$ и имеющая с.-н. йорданов идеал, не являющийся идеалом.

Доказательство основано на простой алгебраической конструкции. Пусть $\mathscr{B}$ - алгебра и $I$ - ее идеал. Пусть существует такой $X \in \mathscr{B} \backslash I$, что $B X \in I$ и $X B \in I$ для всех $B \in \mathscr{B}$. Понятно, что в таком случае алгебра $\mathscr{B}$ не унитальна. Обозначив через $\widetilde{\mathscr{B}}$ ее унитализацию $\mathscr{B}+\mathbb{C} 1$, положим

$$
\mathscr{A}=\left\{\left(\begin{array}{ll}
A & B \\
C & D
\end{array}\right): B, C \in \mathscr{B} \text { и } A, D \in \widetilde{\mathscr{B}}\right\} \text {. }
$$

Пусть, далее,

$$
J=\left\{\left(\begin{array}{cc}
R & S \\
T & U
\end{array}\right)+\nu\left(\begin{array}{cc}
0 & X \\
X & 0
\end{array}\right): R, S, T, U \in I, \nu \in \mathbb{C}\right\} .
$$

Прямой подсчет показывает, что $J$ - йорданов идеал алгебры $\mathscr{A}$, не являющийся, однако, ее идеалом, поскольку

$$
\left(\begin{array}{ll}
1 & 0 \\
0 & 0
\end{array}\right)\left(\begin{array}{cc}
0 & X \\
X & 0
\end{array}\right)=\left(\begin{array}{cc}
0 & X \\
0 & 0
\end{array}\right) \notin J .
$$


Если $\mathscr{B}$ является $C^{*}$-алгеброй, то и $\mathscr{A}$ можно считать $C^{*}$-алгеброй.

Возьмем теперь в качестве $\mathscr{B}$ алгебру $K(H)$. Хорошо известно, что если $I_{1}$ - несепарабельный с.-н. идеал в $\mathscr{B}$, а $I$ - его сепарабельная часть (замыкание множества всех операторов конечного ранга из $\left.I_{1}\right)$, то $\mathscr{B} I_{1} \subset I$ и $I_{1} \mathscr{B} \subset I$. Поэтому в качестве $X$ можно взять любой самосопряженный оператор из $I_{1} \backslash I$. Алгебра $\mathscr{A}$ совпадает с $K(H \oplus H)+\mathbb{C} P+\mathbb{C} 1$, где $P$ - проектор на первое слагаемое в $H \oplus H$, так что $\mathscr{A}$ является коммутативным расширением алгебры $K(H \oplus H)$. Унитарно-инвариантная норма на $J$ естественно индуцируется нормой идеала $I_{1}$.

Заметим, что поскольку идеал $I$ в предыдущей конструкции является сепарабельной частью несепарабельного с.-н. идеала, он (а следовательно, и построенный по нему йорданов идеал $J$ ) не может быть рефлексивным. Наш последний результат показывает необходимость такого геометрического ограничения.

Теорема 9. Если с.-н. йорданов идеал $J C^{*}$-алгебры $\mathscr{A}$ рефлексивен как банахово пространство (в норме $\|\cdot\|_{J}$ ), то он является с.-н. идеалом этой алгебры.

\section{ЛитерАТУРА}

[1] M. Brešar, A. Fošner, M. Fošner, Monatsh. Math., 145 (2005), 1-10. [2] P. Civin, B. Yood, Pacific J. Math., 15 (1965), 775-797. [3] C. K. Fong, G. J. Murphy, Acta Sci. Math., 51 (1987), 441-456. [4] I. N. Herstein, Topics in Ring Theory, The University of Chicago Press, Chicago, 1969. [5] N. Jacobson, C. E. Rickart, Trans. Amer. Math. Soc., 69 (1950), 479-502. [6] E. Kissin, V. S. Shulman, Quart. J. Math., 57:2 (2006), 215-239.

Department of Mathematics and Computer Sciences,

University of Maribor

Поступило в редакцию e-mail: bresar@uni-mb.si 24 декабря 2006 г.

Department of Computing, Communication Technology and Mathematics, London Metropolitan University

e-mail: e.kissin@londonmet.ac.uk

Вологодский технический университет,

кафедра высшей математики

e-mail: shulman_v@yahoo.com

\section{О мере с максимальной энтропией для потока Тейхмюллера на пространстве модулей абелевых дифференциалов*}

(с) 2008. А. И. БУФетов, Б. М. Гуревич

Для целого числа $g \geqslant 2$ и неупорядоченного набора $\kappa=\left(k_{1}, \ldots, k_{m}\right)$ с $k_{i} \in \mathbb{N}$, $k_{1}+\cdots+k_{m}=2 g-2$, обозначим через $\mathscr{M}_{\kappa}$ пространство модулей римановых поверхностей рода $g$, снабженных голоморфными абелевыми дифференциалами $\omega$ с нулями порядка $k_{1}, \ldots, k_{m}$ и с $(1 / 2 i) \int \omega \wedge \bar{\omega}=1$. Пространство $\mathscr{M}_{\kappa}$ не

*Работа первого автора поддержана грантом NSF nо. 0604386 и Фондом Эдгара Оделла Ловетта в университете Райса; работа второго автора поддержана грантами РФФИ 07-0100203 и 07-01-92215-НЦНИЛ. 